\title{
Noruega: desarrollo humano
}

\author{
Claudia Angélica Córdova González*
}

\begin{abstract}
El desarrollo sólo tiene sentido en la medida en que impacta favorablemente a la calidad de vida. El Índice de Desarrollo Humano del Programa de las Naciones Unidas para el Desarrollo integra la esperanza de vida, educación, analfabetismo, pobreza, equidad de género, entre otros. Durante diez años, aunque no de manera consecutiva, Noruega aparece situada en el primer lugar de dicho índice. En este texto se revisa dicha experiencia de alto desarrollo humano.
\end{abstract}

Desarrollo Humano e Índice de Desarrollo

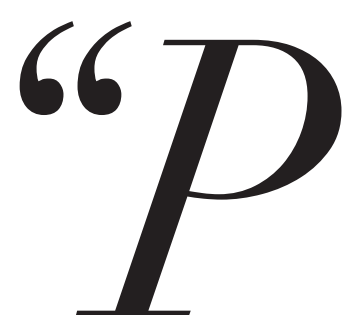

HUMANO

ARA LOGRAR cambios en este sistema en el que hoy nos vemos envuelto, es muy importante el desarrollo humano basado en el aprendizaje del individuo en donde en primera instancia se debe trabajar en crear la filosofía de trabajo, se guíe a la persona, se motive y luego se le dé autonomía para desarrollar su potencial al máximo con diferentes enfo-

*Estudiante de la maestría en Ciencia Política de la Universidad Autónoma de Zacatecas, México. ques, motivando a que sean personas comprometidas e involucradas que agreguen valor a la organización, a sus clientes internos, a las comunidades y a la sociedad; y que al mismo tiempo estos ciudadanos sean responsables por sus acciones y del impacto de sus actividades sobre la sociedad y medioambiente".

En la definición del Programa de Naciones Unidas para el Desarrollo (PNUD), el desarrollo humano es concebido como "la expansión de la libertad de las personas. La libertad es el conjunto de oportunidades para ser y actuar y la posibilidad de elegir con autonomía. Tanto las oportunidades como la participación de los individuos para generarlas están influidas por el entorno en el que viven, y en ese sentido el desarrollo humano es local".? Pudieran desprenderse una variedad de oportunidades, 
pero se centra sobre tres principales: poder tener una vida prolongada y saludable, tener conocimientos que proporciona una buena educación y poder acceder a esos recursos necesarios para tener una vida decente. ${ }^{3}$ En consecuencia, si no tenemos estas oportunidades básicas, difícilmente se alcanzarán otras.

Con la idea de que el subdesarrollo es originado por una falta de capacidades fundamentales, más que y de matriculación escolar (se pondera con un tercio); cada uno de éstos se calcula por medio de la fórmula del índice parcial que anteriormente señalamos, quedando de la siguiente manera:

Índice de educación= $2 / 3$ (índice de alfabetización de adultos) + I/3 (índice de matriculación escolar).

Cuadro i

Tres aspectos integradores del IDH

\begin{tabular}{|c|c|c|c|}
\hline Número & Dimensión & Integración & Valores mínimos y máximos \\
\hline I & Salud & $\begin{array}{l}\text { Medido por medio de la esperanza de } \\
\text { vida al nacer. }\end{array}$ & Mínimo 25 años y máximo 85 años. \\
\hline 2 & Educación & $\begin{array}{l}\text { Se mide como una combinación de } \\
\text { dos terceras partes de la tasa de } \\
\text { alfabetización de adultos y una } \\
\text { tercera parte de la tasa de } \\
\text { matriculación a educación primaria, } \\
\text { secundaria y terciaria. }\end{array}$ & $\begin{array}{l}\text { *Tasa de alfabetización de adultos (se considera a } \\
\text { las personas mayores a i5 años de edad): mínimo o\% } \\
\text { y máximo Ioo\%. } \\
\text { *Tasa de matriculación escolar (se considera a las } \\
\text { personas entre } 6 \text { y } 24 \text { años de edad): mínimo o\% y } \\
\text { máximo ıoo\%. }\end{array}$ \\
\hline 3 & Ingreso & $\begin{array}{l}\text { Medido por el pib per cápita real en } \\
\text { dólares ppc. }\end{array}$ & $\begin{array}{l}\text { pib per cápita real en dólares ppc: mínimo de } \text { SIOO } \\
\text { dólares ppc y máximo de } \$ 40,000 \text { dólares ppc. }\end{array}$ \\
\hline
\end{tabular}

Fuente: Estudios sobre Desarrollo Humano. Programa de las Naciones Unidas para el Desarrollo en México. No. 2003-I.

un problema de ingreso, se crean índices para medir el desarrollo humano. El utilizado en la actualidad es el elaborado por la PNUD, denominado índice de desarrollo humano e incorpora tres elementos básicos: educación, salud e ingreso necesario para tener acceso a bienes y servicios (cuadro I). La metodología de cálculo se ha modificado a lo largo del tiempo. ${ }^{4}$

Hablamos de dimensiones, de elementos integrados y rangos, pero ¡cómo es que lo calculamos? Cada una de las dimensiones es calculada con la siguiente formula:

Índice $\operatorname{parcial}(\mathrm{x})=\operatorname{valor}$ actual $(\mathrm{xi})$ - valor mínimo $(\mathrm{xi})$ valor máximo(xi)-valor mínimo(xi)

Para el caso del cálculo del índice de PIB per capita (y) la fórmula se aplica con logaritmos:

Índice $(y)=\log (y)-\log (y$ min $)$

$\log (y \max )-\log (y \min )$

Como podemos ver en el cuadro i, el índice de educación se compone de dos factores, el de alfabetización de adultos (cuya ponderación es de dos tercios)
Después de que se ha realizado el cálculo de las tres dimensiones, se efectúa un promedio simple de estos tres, y lo obtenemos dividiendo la suma de estos tres entre 3.

$\mathrm{IDH}=($ índice de salud $)+($ índice de educación $)+($ índice de ingreso

Luego de obtener un porcentaje, el PNUD otorga una categoría en base al resultado (cuadro 2).

Cuadro 2.

Los rangos del IDH

\begin{tabular}{lll}
\hline Número & Rangos & $\begin{array}{l}\text { Categoría de Desarrollo } \\
\text { Humano }\end{array}$ \\
\hline $\mathrm{I}$ & $0.89<\mathrm{IDH}<0.99$ & Muy alto \\
\hline 2 & $0.75<\mathrm{IDH}<0.88$ & Alto \\
\hline 3 & $0.58<\mathrm{IDH}<0.74$ & Medio \\
\hline 4 & $0.33<\mathrm{IDH}<0.54$ & Bajo \\
\hline 5 & $0.00<\mathrm{IDH}<0.32$ & Crítico \\
\hline
\end{tabular}

Fuente: Estudios sobre Desarrollo Humano. Programa de las Naciones Unidas para el Desarrollo en México. No. 2003-I. 
El índice de desarrollo humano (IDH) se basa en la creencia de que si el crecimiento económico es un factor importante para darnos una idea del avance de un país, no es el fundamental, es decir, desarrollo y crecimiento son dos conceptos distintos. Para esclarecer lo anterior, pudiéramos decir que algunos países que entran dentro de la medición pueden tener un buen escaño si solamente se considera el ingreso per cápita, pero si le incluimos otros elementos, descienden. $\mathrm{O}$, en caso contrario, países que promueven una buena política social, brinda servicios sociales, pero en cuanto a ingreso se refiere no es el necesario para solventar las necesidades más apremiantes de su población.

Las motivaciones que, sostiene el PNUD, están detrás de los Informes de Desarrollo Humano, son las de diagnosticar problemas y concientizar a los gobiernos para otra gobernanza mundial, en la que se propicie el desarrollo de las capacidades al estilo de las propuestas de Amartya Sen. Se trata de no confiar en el derrame del crecimiento por goteo, sino en llamar la atención en invertir directamente en la gente, en servicios de salud, educación, aumentar la esperanza de vida. ${ }^{5}$

\section{Calidad de Vida}

La calidad de vida, que es lo pretende reflejar el IDH, se compone por elementos objetivos y subjetivos. Los objetivos son palpables, y en cierta forma medibles, como lo es el acceso a salud, educación, esperanza de vida, etcétera. Los segundos tienen más que ver con percepciones personales, que atañen a la felicidad y la satisfacción. Por su carácter, generalmente, son apartados del estudio económico y social. Además nos damos cuenta que la participación del Estado como agente responsable de proveer esta calidad de vida es fundamental. Un ejemplo de esa participación del Estado, en donde se brinda calidad de vida a los ciudadanos, que ocupa el primer lugar dentro de IDH, es un país nórdico, Noruega.

\section{Nociones de Desarrollo Humano en Noruega}

Como lo mencionamos en un principio, en diferentes informes realizados por el PNUD en relación con el desarrollo humano, Noruega figura en primer lugar (aunque no de manera consecutiva) por más de io años dentro del grupo denominado Muy Alto. En los cuadros 3 y 4 se especifica lo anteriormente expuesto.

Cuadro 3

IDH (Muy Alto, considerado país desarrollado).

\begin{tabular}{|c|c|c|c|c|}
\hline \multicolumn{5}{|l|}{ Posición } \\
\hline $\begin{array}{l}\text { Informe } \\
2 \mathrm{OII}\end{array}$ & $\begin{array}{l}\text { Variación } \\
\text { respecto al } \\
\text { Informe } \\
20 \text { o }\end{array}$ & País & $\mathrm{IDH}$ & $\begin{array}{l}\text { Variación } \\
\text { respecto al } \\
\text { Informe } \\
20 \text { o }\end{array}$ \\
\hline I & Igual & Noruega & $0.94^{3}$ & $\begin{array}{l}\text { Aumento } \\
(\text { o.oo5 })\end{array}$ \\
\hline
\end{tabular}

Fuente: Programa de las Naciones Unidas para el Desarrollo, Informe sobre Desarrollo Humano (2010 y 2011).

Cuadro 4

Primer lugar en el IDH por año.

\begin{tabular}{cccc}
\hline Año & País & Año & País \\
\hline 2011 & Noruega & 2005 & Islandia \\
\hline 2010 & Noruega & 2004 & Noruega \\
\hline 2009 & Noruega & 2003 & Noruega \\
\hline 2008 & Noruega & 2002 & Noruega \\
\hline 2007 & Noruega & 2001 & Noruega \\
\hline 2006 & Islandia & 2000 & Noruega
\end{tabular}

Fuente: Programa de las Naciones Unidas para el Desarrollo, Informe sobre Desarrollo Humano (2006, 2007, 2008, 2009, 20I0 у 20II).

Dentro de los rubros más sobresalientes encontramos los siguientes, que son los que permiten que la calidad de vida y desarrollo humano sea una realidad.

\section{a) Pleno empleo y seguridad social}

En 2010, el 30\% de los empleados se ubica en el sector público, convirtiéndolo en uno de los países a nivel internacional con mayor población en este sector. ${ }^{6} \mathrm{Al}$ verificar las actividades económicas realizadas por 
personas de entre 15 a 74 años se puede decir que $62 \%$ tiene dedicación de tiempo completo o parcial, $15 \%$ está en proceso de formación y $2 \%$ está realizando trabajos en casa.

Noruega cuenta con un servicio de salud pública universal y un plan de seguridad social nacional para todos los ciudadanos y residentes. Existe un permiso por baja maternal o paternal, pagada al Ioo\% durante 46 semanas o con $80 \%$ si se incrementa a 56 semanas, lo que permite que se dé una combinación entre el trabajo y el cuidado. Los cuidados pueden ser combinados entre los dos padres, para que los papás puedan establecer lazos más fuertes desde el nacimiento.

\section{b) Educación}

La educación, y el sistema educativo, es una tarea que asume el Estado por medio del Ministerio de Educación e Investigación, basado en la herencia cristiana, humanista, con respeto a la dignidad humana y la naturaleza, la libertad intelectual, igualdad y solidaridad, valores que surgen de la diversidad religiosa y creencias y que tienen como fiel soporte los derechos humanos. Se promueve la democracia, igualdad y el pensamiento científico. La educación se encamina para pulir capacidades, dar conocimientos, forjar habilidades para participar activamente en actividades laborales y ayudar a la sociedad en la que se desenvuelve de una manera crítica, ética y con conciencia ambiental.7 El Ministerio tiene a su cargo la educación primaria, secundaria, superior y la investigación. Su ser y su actuar están estipulados en Reglamento de la Ley de educación vigente desde 2006.

\section{c) Industria y manejo de los recursos naturales}

Representa un enorme reto para esta nación el manejo de sus recursos naturales, ya sea por el clima extremo, mares bravos, inviernos largos, terrenos ásperos. Es cuando ven la importancia de desarrollar formas, conocimientos y técnicas que les ayuden a explotarlos al máximo. Es el caso del petróleo. Esta actividad es offshore (en alta mar), realizada desde hace 40 años en el Mar del Norte, Mar Noruego y Mar de Barents, y es el Estado quien controla la explotación de este recurso. ${ }^{8} \mathrm{Y}$ todos aquellos externos e internos que rea- lizan inversión en este rubro deben de adaptarse a las leyes y regulaciones que no están en negociación y pagan $78 \%$ de sus utilidades como impuesto al Estado. Las empresas privadas son buscadas como socios, más que verlos como rivales o enemigos, ya que a raíz de estas relaciones han aprendido el negocio, y se volvieron más independientes de ellas. Asimismo, se tiene la facultad por ley de verificar todo lo que hacen las compañías, y si existiese algún problema se resuelve en el mismo país. ${ }^{9}$

\section{Conclusiones}

Para concluir, pudiéramos decir que existen diversos tipos de indicadores que nos servirían para medir la calidad de vida de un país, pero consideramos que el Índice de Desarrollo Humano contiene elementos básicos para determinar el desarrollo humano. Pensamos que para el caso de otros países, los elementos integradores son subjetivos y variantes por lo que nos darían resultados inciertos, lo que no permitiría tomar decisiones correctas en cuanto a políticas públicas encaminadas a esa calidad de vida tan anhelada. Sin embargo, entendemos que por algún lugar tenemos que partir para desarrollar indicadores más acertados que nos viertan propuestas más acertadas.

Noruega es un ejemplo a nivel internacional de que es posible tomar decisiones correctas y de que el trabajo en equipo entre Estado, sociedad y sector privado es importante para prosperar como nación. En un sistema capitalista en el que cada vez se hace más amplia la brecha de desigualdad e injusticia, debemos de proponer modelos dentro de este sistema que sean más justos con la sociedad.

Si no se cuenta con el conocimiento ni tecnología podemos ver como alternativa la experiencia de Noruega consistente en la participación del sector privado e inversiones extranjeras en empresas paraestatales que las dotan del conocimiento con el que no cuentan y que es indispensable para el uso y manejo correcto de sus recursos naturales, pero esta participación no es de apertura amplia, sólo la necesaria para beneficiarse mutuamente. El desarrollo de ciencia y tecnología no se queda atrás, ya que es incentivada por el Estado, pues existe el reconocimiento de que de ello depende el futuro del mismo. 


\section{$-\operatorname{notas} \cdot-$}

'Nayezkha Flores y Rafael Rodríguez, "Crecimiento económico y desarrollo humano", Observatorio Laboral Revista Venezolana, vol. 4, núm. 7, enero-junio, 20II, pp. 55-7o, Universidad de Carabobo, Venezuela.

Índice de Desarrollo Humano (20II), Programa de las Naciones Unidas para el Desarrollo. Edición y producción, Communications Development Incorporated, ciudad de Washington.

${ }^{3}$ Teresa Torres Solé y Rafael Allepuz Capdevila, "El desarrollo humano: perfiles y perspectivas futuras", Estudios de Economía Aplicada, vol. 27, núm. 2, agosto, 2009, pp. 545-56ı, Asociación Internacional de Economía Aplicada, España.

4López-Calva y Vélez Grajales, "El concepto de desarrollo humano, su importancia y aplicación en México", Estudios sobre Desarrollo Humano. PNUD México, no. 2003-г. Octubre, 2003.

${ }^{5} \mathrm{~L}$. Coraggio (2009). "La economía social y solidaria ante la continua insistencia en el desarrollo económico", Memorias del primer seminario internacional de desarrollo económico y ca- lidad de vida. Bogotá D.C., Colombia, Universidad la Gran Colombia, Facultad de Ciencias Económicas y Administrativas.

${ }^{6}$ Instituto Nacional de Estadística a cargo del Ministerio de Asuntos Exteriores (2012), Minidatos de Noruega, http://www. ssb.no/, Oslo, marzo. Recuperado I5 de octubre 2012.

- Embajada de Noruega en México (2013), http://www.noruega.org.mx/News_and_events/Noruega_tP_AnDNT, México. Consultado el 24 de enero 2013.

${ }^{8}$ Embajada de Noruega en México (2013), http:/www.noruega.org.mx/News_and_events/Noruega_UP_AMDINT, México. Consultado el 24 de enero 2013.

9OilProduction.net (2010), El exitoso modelo de extracción de Noruega, http://www.oilproduction.net/cms/index. php?option $=$ com_content $\&$ view $=$ article $\& i d=798$ :el-exitosomodelo-de-extraccion- de-noruegadcatid=53: notas - de opinion\&Itemid= =II, Ecuador, febrero, consultado el 28 de octubre del 2012. 

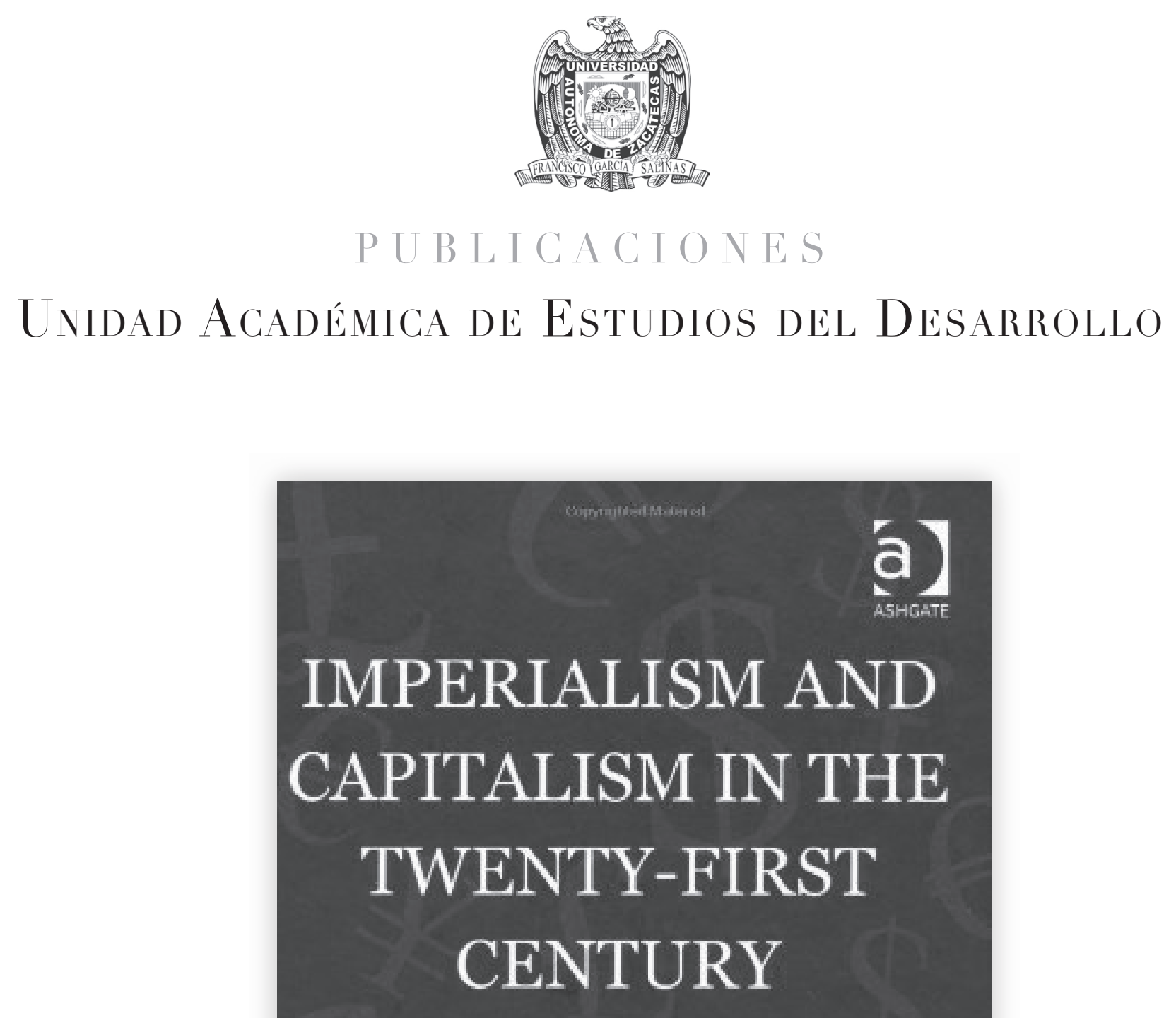

A System in Crisis

James Petras and Henry Veltmeyer 